\title{
ProtecT-ing against prostate cancer
}

Initial results for the Prostate testing for cancer Treatment (ProtecT) randomized phase III trial have recently been reported in The Lancet Oncology. The ProtecT trial is the largest ongoing randomized controlled trial of prostate cancer treatment in the world, and it aims to investigate the effectiveness of radical prostactectomy, radiotherapy or active monitoring for men with localized prostate cancer.

The study recruited participants through 337 primary care centres in nine cities around the UK. In total, 228,966 men aged 50-69 years were invited to take part in the trial between 2001 and 2009. Of these men, $44 \%$ attended their initial appointment and 82,429 underwent a serum PSA test. Threshold serum PSA level for an offer of biopsy was set at $\geq 3.0 \mathrm{ng} / \mathrm{dl}$ and applied to $11 \%$ of patients who had their serum PSA tested. Prostate biopsy was performed on 7,414 men and 2,896 men were subsequently diagnosed with prostate cancer.

\section{4 ...ProtecT trial is the largest ... controlled trial of prostate cancer treatment in the world... 77}

Of these patients diagnosed with prostate cancer, 2,417 had localized disease and were eligible to continue in the trial. A further 247 participants were recruited from the Cluster randomized triAl of PSA testing for Prostate cancer (CAP) trial, between 2001 and 2009, giving a total of 2,664 patients who were eligible for random assignment, of which $62 \%$ agreed to participate. Men were randomly assigned to active monitoring, radiotherapy or surgery in a ratio of 1:1:1 with 545, 545 and 553 recruits in each group, respectively. Participants in this trial were predominantly white and married; the median age of patients was 58 years.

A positive correlation between serum PSA concentration, age and receipt of biopsy was shown. In particular, the relationship between serum PSA concentration and cancer diagnosis was unchanged on age adjustment, but adjustment for serum PSA attenuated the relationship between age and diagnosis.

ProtecT was designed to address key issues in major conventional treatments for localized prostate cancer. The primary patient outcomes are probable or definite cancer-specific mortality and secondary outcomes include overall mortality, incidence of metastases, disease progression, treatment complications and cost effectiveness at a follow-up period of 10 years. Data on the effectiveness of the tested treatments will be provided in 2016.

Louise Stone

Original article Lane, A. J. et al. Active monitoring, radical prostatectomy, or radiotherapy for localised prostate cancer: study design and diagnostic and baseline results of the ProtecT randomised phase 3 trial. Lancet Oncol. doi:10.1016/S1470-2045(14)70361-4 